\title{
Keeping von Willebrand Factor under Control: Alternatives for ADAMTS13
}

\author{
Claudia Tersteeg, $\mathrm{PhD}^{1}$ Rob Fijnheer, MD, $\mathrm{PhD}^{2}$ Gerard Pasterkamp, MD, $\mathrm{PhD}^{2}$ \\ Philip G. de Groot, $\mathrm{PhD}^{2}$ Karen Vanhoorelbeke, $\mathrm{PhD}^{1}$ Steven de Maat, MSc ${ }^{2}$ Coen Maas, $\mathrm{PhD}^{2}$
}

\author{
${ }^{1}$ Laboratory for Thrombosis Research, IRF Life Sciences, KU Leuven \\ Campus Kulak Kortrijk, Kortrijk, Belgium \\ 2 Laboratory of Clinical Chemistry and Hematology, UMC Utrecht, \\ Utrecht, The Netherlands
}

Address for correspondence Coen Maas, PhD, Laboratory of Clinical Chemistry and Hematology, UMC Utrecht, Heidelberglaan 100, G.03.550, 3584 CX Utrecht, the Netherlands (e-mail: cmaas4@umcutrecht.nl).

Semin Thromb Hemost 2016;42:9-17.

\begin{abstract}
Keywords

- ADAMTS13

- plasmin

- VWF

- thrombotic thrombocytopenic purpura

- thrombotic microangiopathy

Von Willebrand factor (VWF) is one of the most important proteins of the hemostatic system. Its multimeric state is essential for its natural function to guide platelets to sites of injury. ADAMTS13 is the key protease that regulates the multimeric state of VWF. Without ADAMTS13, VWF multimers can grow to pathologically large sizes. This is a risk factor for the life-threatening condition thrombotic thrombocytopenic purpura (TTP). In this condition, VWF-rich thrombi occlude the microvasculature of various tissues. Intriguingly, a complete ADAMTS13 deficiency does not cause continuous TTP, either in patients or genetically targeted mice. Instead, TTP occurs in episodes of disease, separated by extended periods of remission. This indicates that regulating factors beyond ADAMTS13 are likely involved in this pathologic cascade of events. This raises the question of what really happens when ADAMTS13 is (temporarily) unavailable. In this review, we explore the possible role of complementary mechanisms that are capable of modifying the thrombogenic potential of VWF.
\end{abstract}

When the vessel wall ruptures during injury or cardiovascular events, platelets are the first to arrive on the scene. These platelets are guided to the damaged vessel wall by von Willebrand factor (VWF), which acts as molecular velcro. The multimeric state and conformation of VWF are key to its platelet-binding properties and enable platelets to bind to exposed subendothelium under the strong shear forces of flowing blood. ${ }^{1}$ The platelet-binding properties of VWF need to be kept under constant control to prevent spontaneous intravascular platelet clustering, which can have dramatic pathologic consequences. When intravascular platelet clustering goes unchecked at sites of high shear forces, the microvasculature can become occluded by microthrombi. ${ }^{2}$ Subsequently, organs rich in microvessels can become ischemic and damaged. The disease thrombotic thrombocytopenic purpura (TTP) is associated with waves of promiscuous platelet-clustering activity of VWF. These attacks of TTP are accompanied by hemolytic anemia and thrombocytopenia.

published online November 23, 2015
Issue Theme Editorial Compilation I; Guest Editors: Emmanuel J. Favaloro, PhD, FFSc (RCPA), and Giuseppe Lippi, MD.

\section{ADAMTS13}

The principal enzyme responsible for controlling the plateletbinding properties of VWF is ADAMTS13 (a disintegrin and metalloproteinase with a thrombospondin type I motif, member 13). Before its identification, it was reported that a VWF-cleaving enzyme is constitutively active in plasma. ${ }^{3}$ This enzyme cleaves VWF in its A2 domain at a $\mathrm{Tyr}_{1605}-\mathrm{Met}_{1606}$ bond (numbering includes the VWF propeptide, relating to positions 842-843 in mature VWF monomers). The VWFcleaving capacity of this enzyme is dependent on metal-ions and promoted by conformational changes in VWF. In its globular form, VWF is resistant to proteolysis, while unfolding facilitates cleavage. ${ }^{4}$ Later studies identified this enzyme as ADAMTS13. 5,6 The molecular mechanisms by which ADAMTS13 interacts with VWF to induce cleavage have been investigated in great detail and show us a unique, elegant, and tightly regulated process. ${ }^{7}$ After VWF undergoes
Copyright @ 2016 by Thieme Medical Publishers, Inc., 333 Seventh Avenue, New York, NY 10001, USA.

Tel: +1(212) 584-4662.
DOI http://dx.doi.org/ 10.1055/s-0035-1564838. ISSN 0094-6176. 
its conformational changes, ADAMTS13 binds to its substrate in multiple steps, positioning itself with high precision for cleavage of the $\operatorname{Tyr}_{1605}-\mathrm{Met}_{1606}$ scissile bond.

\section{Thrombotic Thrombocytopenic Purpura}

Lowered or absent ADAMTS13 activity can result in circulating VWF multimers that are unusually large. These VWF multimers are capable of spontaneous platelet recruitment in the circulation, especially under conditions of high shear. This can ultimately lead to the clinical syndrome of TTP. Reduced ADAMTS13 activity can be caused by a rare genetic deficiency (Upshaw-Shulman syndrome) but is more often the result of inhibitory autoantibodies (acquired TTP). TTP attacks are characterized by hemolytic anemia and thrombocytopenia, and are often accompanied by neurologic abnormalities, fever, and renal failure (although involvement of kidneys is less than in other forms of thrombotic microangiopathy ${ }^{2}$ ). The obstructive microthrombi are held responsible for these diverse pathologic features. If left untreated, TTP leads to organ failure with lethal consequences in the large majority of cases.

Plasma exchange and immunosuppressive therapy are valuable in TTP caused by an acquired ADAMTS13 deficiency. However, exciting developments may lead to more therapeutic options in the near future. First, recombinant forms of ADAMTS13 are currently under development, ${ }^{8,9}$ some of which are resistant against neutralizing autoantibodies. ${ }^{10}$ The common aim of these therapies is to restore ADAMTS13 activity in patients, which, in turn, should facilitate cleavage of VWF to destroy VWF-rich microthrombi. Furthermore, there is evidence that VWF multimer size can be pharmacologically targeted with $N$-acetylcysteine (NAC). This Food and Drug Administration (FDA)-approved drug is (among others) useful for treatment of acetaminophen toxicity and chronic obstructive lung disease. Interestingly, NAC has been shown to be able to reduce disulfide bonds in VWF, reducing size and activity of VWF multimers. Case reports on the treatment of TTP with NAC describe varying outcomes. ${ }^{11,12}$ A clinical trial is currently ongoing to test its efficacy under controlled conditions (ClinicalTrials.gov Identifier: NCT01808521). Finally, baboon experiments previously demonstrated that inhibition of the platelet GPIb-VWF interaction with a nanobody (camelid single-chain antibody) is an effective and safe strategy for managing acquired TTP. ${ }^{13,14}$ A clinical trial has been performed (TITAN Trial; ClinicalTrials.gov Identifier: NCT01151423) to investigate the therapeutic potential of VWF-targeting nanobody caplacizumab (ALX-0681) in conjunction with plasma exchange for treatment of TTP. ${ }^{15}$ The first published results from this trial are very promising: nanobody treatment reduces the time to platelet count normalization and reduces the need for plasma exchange. ${ }^{16}$

\section{The Mysterious Erratic Nature of TTP}

\section{Lessons from Patients}

During attacks of acquired TTP, ADAMTS13 activity is generally lower than it is during remission in the same patients. ${ }^{17}$ Variations in autoantibody levels may help explain this variation in ADAMTS13 activity: when autoantibody titers against ADAMTS13 are high, its activity decreases. ${ }^{18}$ Based on the attack/remission clinical phenotype seen in TTP, it is attractive to hypothesize that ADAMTS13 activity levels need to remain above a threshold for the maintenance of a healthy perfusion of the microvasculature and prevention of TTP. ${ }^{19}$ However, several lines of evidence argue against this concept: (1) patients with acquired TTP can have undetectable ADAMTS13 activity while being in remission and (2) patients with congenital ADAMTS13 do not experience continuously ongoing TTP but develop attacks of TTP in a pattern that closely resembles that of acquired TTP patients. Indeed, despite their complete congenital ADAMTS13 deficiency, such patients can remain completely asymptomatic for years. ${ }^{20-22}$

\section{Lessons from Mouse Models}

The findings from congenital ADAMTS13-deficient patients have been corroborated in congenitally deficient ADAMTS13deficient mice: these mice also do not spontaneously develop symptoms of TTP nor experience continuous thrombotic microangiopathy. ${ }^{23}$ They only develop features that approximate TTP after exposure to pathogenic toxins, ${ }^{23}$ or after injection with large amounts of recombinant multimeric $\mathrm{VWF}^{9}$ Intriguingly, congenital ADAMTS13 deficiency alone is not sufficient to increase the susceptibility of these mice for these triggers. TTP-like symptoms mainly develop in ADAMTS13-deficient mice with a very specific genetic background (i.e., CASA/Rk), but not in ADAMTS13 deficient mice with other genetic backgrounds. This strongly indicates that additional genetic risk factors modulate the capacity of these mice to develop TTP-like symptoms. Although this strain produces more VWF than the other strains, this is not held responsible for the heightened sensitivity of these mice to develop TTP-like symptoms. ${ }^{23}$ The identification of additional genetic risk factors in the CASA/Rk strain may have significant impact on our understanding of TTP.

These combined observations from human TTP patients, as well as mice, strongly suggest that ADAMTS13 deficiency is an important risk factor for the development of TTP. However, it is also clear that additional factors modulate the disease phenotype.

\section{A Second Hit for TTP}

As ADAMTS13 deficiency alone cannot explain the onset of all TTP attacks, it is probable that a triggering event is needed. It is attractive to hypothesize that an infection constitutes this trigger. In the case of Shiga toxin-mediated hemolytic uremic syndrome (ST-HUS), another form of thrombotic microangiopathy, the responsible pathogens have been identified. These are Shigella dysenteriae type 1 and certain strains of Escherichia coli, which produce shigatoxin. ${ }^{24}$ Exposure to these pathogens via food intake can trigger severe thrombotic microangiopathy, independent of ADAMTS13 activity. On a macroscopic level, ST-HUS is clinically more commonly associated with acute kidney injury than TTP. ${ }^{2}$ On a microscopic level, analyses of the composition of obstructive microthrombi reveal the involvement of fibrin in ST-HUS, whereas 
microthrombi in TTP are (enigmatically) fibrin-poor. ${ }^{25}$ These differences between ST-HUS and TTP suggest that, if there is a pathogen is responsible for triggering TTP, it may be different from the pathogens that cause ST-HUS.

There is scientific evidence that links infection to TTP: recent studies showed that acute TTP attacks are associated with elevated levels of circulating DNA-histone complexes, as well as other components that can be traced back to release by neutrophils. ${ }^{26}$ These neutrophil-derived components are able to trap bacterial pathogens and assist in their destruc$\operatorname{tion}^{27}$ and were originally discovered in severe bacterial septicemia. ${ }^{28}$ Their identification in TTP leads to the attractive hypothesis that these DNA-histone complexes, rather than the underlying infection, constitute the second hit that elicits an attack. However, are the DNA-histone complexes that are seen in TTP really caused by infection or are they a consequence of thrombotic microangiopathy? This same study shows neutrophil-released components in several other forms of thrombotic microangiopathy (unrelated to ADAMTS13 deficiency). ${ }^{26}$ Furthermore, neutrophil release products have been reported in a variety of prothrombotic states that are not directly linked to infection, ${ }^{29-33}$ and are released during ex vivo storage of red blood cells. ${ }^{34}$ TTP attacks are often associated with hemolytic anemia, resulting from shear-related cellular damage to red blood cells at sites of vascular occlusions. It is attractive to speculate that in TTP neutrophils undergo a fate that is similar to that of erythrocytes, resulting in the release of their contents in a shearrelated, but infection-unrelated, manner. This process, in turn, could worsen TTP symptoms by providing a "second hit" that reinforces microthrombosis. ${ }^{35}$

\section{Assistance for ADAMTS13 to Keep VWF Activity "Under Control"}

It is evident that ADAMTS13 is a central physiologic regulator of VWF multimer size in blood. However, is it also certain that ADAMTS13 is the only protein that regulates the thrombogenicity of VWF? The proverb "Amicus certus in re incerta cernitur" by the roman poet Quintus Ennius (239 BC-c. 169 BC: http://en.wikipedia.org/wiki/Ennius) can be popularly translated as "A friend in need is a friend indeed." The observation that congenital ADAMTS13-deficient persons and mice can be symptom free for extended periods of time suggests that ADAMTS13 may get help to keep VWF activity under control.

\section{The Complement System}

In complement-mediated thrombotic microangiopathy, excessive activation of the complement system leads to thrombus formation. Mutations in factors $\mathrm{H}$ and $\mathrm{I}$, which together control the alternative pathway of the complement system, are associated with this disease. ${ }^{36,37}$ The complement system influences VWF release and function on multiple levels. First, unregulated formation of membrane-attack complexes causes endothelial cell damage and platelet activation. This can trigger VWF release, which may contribute to microthrombosis. Second, endothelial-cell tethered platelet-VWF complexes can recruit and activate components of the alternative complement pathway. ${ }^{38}$ Under normal conditions, this is kept under tight control by factors I and $\mathrm{H}$ (which can directly bind to VWF). ${ }^{38,39}$ Third, factor $\mathrm{H}$ may directly influence the cleavage of VWF by ADAMTS13. A recent study showed that factor $\mathrm{H}$ enhances the cleavage of soluble VWF by ADAMTS13, ${ }^{39}$ while another study showed that VWF cleavage by ADAMTS13 was inhibited by factor $\mathrm{H}$ on the surface of endothelial cells. $^{40}$

Finally, detailed biochemical studies identified a direct influence of factor $\mathrm{H}$ on VWF that is unrelated to its role in the complement system: factor $\mathrm{H}$ directly modifies multimeric VWF in a nonenzymatic manner through reduction in large soluble multimers. ${ }^{41}$ Together, these studies offer an explanation for the genetic association between factor $\mathrm{H}$ mutations and (complement-mediated) thrombotic microangiopathy: factor $\mathrm{H}$ has multiple protective roles, which help control the thrombogenicity of VWF. It is important to note that this form of thrombotic microangiopathy is largely unrelated to ADAMTS13 deficiency. $^{2}$

\section{Enzymes}

A variety of enzymes can cleave VWF in vitro. These enzymes include thrombin and plasmin, ${ }^{42}$ as well as cathepsin G, neutrophil elastase, proteinase 3, matrix metalloproteinase $9,{ }^{43}$ and granzyme $\mathrm{M}$ and $\mathrm{B} .{ }^{44}$ The molecular mechanisms by which these enzymes cleave VWF are not uniform. Though some enzymes target the $\mathrm{Tyr}_{1605}-\mathrm{Met}_{1606}$ bond in VWF A2 domain (which is represented in the fluorogenic FRETS-VWF73 peptide ${ }^{45}$ ) that is also targeted by ADAMTS13, other enzymes have been shown to target different sites. Most of the reported enzymes can be secreted in active form by circulating cells or become activated in blood plasma during clot formation or breakdown. It is attractive to hypothesize that these enzymes contribute to the proteolysis of VWF in vivo and have protective roles during attacks of TTP.

\section{Neutrophil Enzymes}

Neutrophils release enzymes during their activation. These enzymes can cleave VWF close to or at the same position in the A2 domain as ADAMTS13, even in the presence of physiological inhibitors. ${ }^{42,43}$ Neutrophil-release products are seen during attacks in various forms of thrombotic microangiopathy ${ }^{26}$ and correlate well with disease activity. ${ }^{46}$ It is attractive to hypothesize that neutrophil enzymes are released for the degradation of VWF in obstructive microthrombi. However, experimental evidence points out that neutrophil enzymes are not (continuously) involved in the degradation of VWF: neutrophil depletion in Adamts13-/- mice does not affect VWF multimer composition. ${ }^{47}$ Taken together, the overall contribution of neutrophil activation to acute TTP episodes appears ambivalent: on the one hand, the release of NETs may provide a "second hit" that worsens microthrombosis, on the other hand, neutrophil enzymes may help ADAMTS13 degrade VWF during microangiopathy. 


\section{Granzymes}

Granules of cytotoxic lymphocytes contain a collection of secretable enzymes named granzymes. Through a precise mechanism that is dependent on formation of immunologic synapses and perforin, granzymes are transferred to compromised target cells to cleave caspases and initiate apoptosis. Granzymes are also produced by other cell types, such as mast cells, that do not have this sophisticated cell-targeting capacity. In this case, granzymes are secreted into the extracellular compartment after degranulation. One of these is granzyme $\mathrm{B}$, which can cleave extracellular matrix proteins, as well as fibrinogen and $\mathrm{VWF}^{48}$ It cleaves VWF effectively when it is unfolded and destroys its platelet-binding capacity. In contrast, granzyme M cleaves VWF in its globular conformation. This does not affect its platelet-binding capacity but instead abrogates the capacity of VWF to carry coagulation factor VIII. ${ }^{49}$ The cleavage sites for both granzymes in VWF differ from each other, explaining the selectivity of their actions on VWF. Granzymes have been implicated in a variety of inflammatory conditions, ${ }^{50}$ where they may modulate the activities of $\mathrm{VWF}^{44}$

\section{Thrombin}

The coagulation cascade generates thrombin at sites of vessel injury. This versatile enzyme is a key regulator of both primary and secondary hemostasis. In a side-by-side comparison of multiple VWF-cleaving enzymes, thrombin has a modest capacity to cleave VWF in comparison to neutrophil elastase. ${ }^{42}$ Nonetheless, these same studies revealed that purified thrombin removes adherent platelets from a collagen surface. This experiment suggests that thrombin modulates platelet adhesion to collagen by cleaving VWF, effectively overruling the well-known platelet-activating properties of thrombin. ${ }^{42}$ This leads to the question: could thrombin influence the role of VWF in TTP?

Elevated thrombin-antithrombin (TAT) complexes, prothrombin fragment F1 +2 , and D-dimer levels are seen in ST-HUS. These markers indicate prothrombin activation that may be attributed to tissue factor expression. ${ }^{51,52}$ The direct thrombin inhibitor hirudin prevents the lethal effects of Shiga toxin in a canine model for ST-HUS, ${ }^{53}$ suggesting that the overall role of thrombin in HUS is pathologic. However, the role of thrombin in TTP is less clearly defined. Similar to HUS, case reports of acute TTP patients describe elevated plasma markers for thrombin activity. ${ }^{52,54-56}$ Paradoxically, this does not appear to lead to fibrin-rich thrombi: microthrombi observed in TTP pathology are generally classified as platelet and VWF-rich, but poor in fibrin. ${ }^{25}$ This remarkable absence of fibrin from these thrombi suggests that thrombin activity in TTP is limited (if present at all). However, these findings certainly do not rule out the possibility that thrombin directly modulates the thrombogenicity of VWF during TTP attacks.

\section{Plasmin}

Plasmin is essential for fibrinolysis (i.e., the breakdown of fibrin polymers in blood clots). The liver produces plasminogen as precursor, which can be activated into plasmin by tissue-type plasminogen activator (tPA). This enzyme mainly becomes effective as an activator of plasminogen in the presence of fibrin. ${ }^{57}$ Both tPA and plasminogen itself bind to fibrin polymers in a lysine-dependent manner, facilitating the enzymatic crosstalk required for successful clot breakdown.

Through an alternative mechanism, plasminogen activation can be triggered on the surface of cells by urokinase-type plasminogen activator (uPA). This occurs in a receptormediated mechanism and does not require the presence of fibrin. The uPA receptor ( $\mathrm{UPAR}$ ) is present on endothelial cells, certain types of leukocytes (e.g., neutrophils), and platelets. ${ }^{58}$ Furthermore, a large number of cellular receptors for plasminogen have been identified, which are thought to direct the multitude of diverse functions that plasmin is thought to execute. ${ }^{59}$ Plasminogen activator inhibitor-1 (PAI-1) is an important regulator of plasminogen activation. This serine protease inhibitor can rapidly bind and inactivate both uPA and tPA, preventing plasmin formation. In similar manner, plasmin is inhibited by $\alpha_{2}$-antiplasmin. When plasmin is bound to fibrin or to a receptor at the cell surface, it is temporarily shielded from inactivation, allowing plasmin to act longer on the cell surface than it would in solution.

Besides its role in fibrinolysis, plasmin can also cleave $\mathrm{VWF}^{42,60,61}$ as well as ADAMTS13, ${ }^{62,63}$ and is able to disaggregate platelet-VWF complexes in the absence of ADAMTS13 in vitro. ${ }^{42}$

\section{Plasmin in TTP: Saint or Criminal?}

We recently explored the possibility that plasmin plays an important protective role in the pathogenesis of TTP by degradation of VWF in microthrombi. ${ }^{64}$ We confirmed that plasmin can cleave VWF and is capable of breaking down platelet-VWF complexes in vitro. These findings support the proposed protective function of plasmin during attacks of thrombotic microangiopathy. However, our findings in patients with TTP are more complex to interpret. In our studies, we found that levels of plasmin- $\alpha_{2}$-antiplasmin (PAP) complexes (representing recent plasminogen activation) are mainly elevated in plasma samples of patients during acute attacks of TTP. In detail, levels of PAP complexes correlate with the extent of thrombocytopenia, which serves as a marker for disease activity as low platelet counts are seen as a consequence of consumptive microthrombosis. This observation appears counterintuitive: if plasmin mediates degradation of microthrombi, why is plasminogen activation seen during active disease, exactly when microthrombi are not sufficiently cleared?

Acute TTP attacks are associated with increased tPA levels, as well as increased PAI- 1 levels. ${ }^{65}$ This could be the result of endothelial cell activation. Surprisingly, fibrin degradation product (FDP) and D-dimer levels remain normal, which indicates that fibrin degradation is low or absent during attacks. It is attractive to hypothesize that plasmin is formed by tPA on platelet-VWF complexes. ${ }^{66}$ However, we found that these complexes do not stimulate tPA-mediated plasminogen activation, ${ }^{64}$ leaving them intact in the combined presence of tPA and plasminogen. In contrast, we found that endothelial 
cells can swiftly generate sufficient amounts of plasmin (via the urokinase system) to break down nearby platelet-VWF complexes under shear flow. Hence, we believe that this latter mode of plasminogen activation takes place during TTP attacks. This leads to the question: how is the urokinase system activated during TTP attacks? Plasminogen activation on the endothelium can be triggered by hypoxia ${ }^{67,68}$ and takes place on the endothelium of ischemic tissue during thrombotic microangiopathy: postmortem kidney sections demonstrate that UPAR expression is highly increased in the kidney vasculature of TTP patients, compared with control subjects. ${ }^{69}$ We therefore propose plasminogen activation in TTP is triggered only after microthrombotic vascular obstructions trigger endothelial cell activation ( - Fig. 1). This plasmin subsequently degrades VWF aiming to eliminate these obstructions, but it may also cause collateral damage by degrading ADAMTS13 when its activity is improperly controlled. ${ }^{62}$ This helps to explain why plasminogen activation will coincide with TTP attacks, but why is this enzyme is not always fully capable of averting disease?

Clinical evidence from patients provides an important lead: acute TTP episodes are accompanied by decreased uPA levels, indicating that this plasminogen activator is being consumed. ${ }^{65}$ We hypothesize that in a situation where obstructive microthrombi are successfully cleared by plasmin, clinical symptoms will not become evident (i.e., the patient will stay in an apparent remission state). However, when the endogenous capacity for plasmin formation becomes exhausted (e.g., after prolonged formation of microthrombi), obstructions are no longer sufficiently cleared with pathologic results. This concept may extend beyond TTP: there is evidence to suggest that an imbalanced plasminogen activation system also has dangerous consequences for other forms of thrombotic microangiopathy. First, plasminogen activation is reported to take place in patients with ST-HUS. ${ }^{51}$ Recent studies identified that genetic variations in the plasminogen gene that reduce its expression form an important independent genetic risk factor for this disease, ${ }^{70}$ indicating a protective function of plasmin against HUS. In conclusion, we (as well as others ${ }^{71}$ ) believe that plasmin can act as an enzymatic "last resort" for the removal of vascular obstructions by cleaving fibrin, VWF, or both.

\section{A Possible Therapeutic Opportunity: Thrombolysis for TTP Treatment}

Bypassing Autoantibodies in TTP: A Clinical Challenge Autoantibodies against ADAMTS13 form the underlying cause for ADAMTS13 deficiency in most patients with TTP. These antibodies also complicate plasma transfusion therapy by neutralizing exogenous ADAMTS13. Based on our recent findings, we propose that endogenous plasmin acts as a natural "emergency replacement" for ADAMTS13. If this is the case, thrombolytic agents may have therapeutic value for patients with TTP. These drugs would help break down obstructive microthrombi but would not be neutralized by autoantibodies.

\section{Proof-of-Concept Experiments}

To explore the hypothesis that thrombolytic agents may be therapeutic in TTP, we administered a single dose of the direct-acting thrombolytic agent streptokinase to ADAMTS13-deficient mice that had been subjected to a model for TTP (via injection of a high dose of recombinant VWF). ${ }^{64}$ We found that his treatment attenuated symptoms of TTP and corrected the thrombocytopenia.

\section{Safety Aspects}

Bleeding is the most well-known side effect of thrombolytic treatment. The risk of thrombolysis-related bleeding is strongly associated with anticoagulant therapy (that many of these patients use) and increases steeply with age. ${ }^{72}$ In these patients, thrombocytopenia is a contraindication for the use of thrombolytic agents. Intuitively, it can be assumed that there will be a severe bleeding risk associated with the

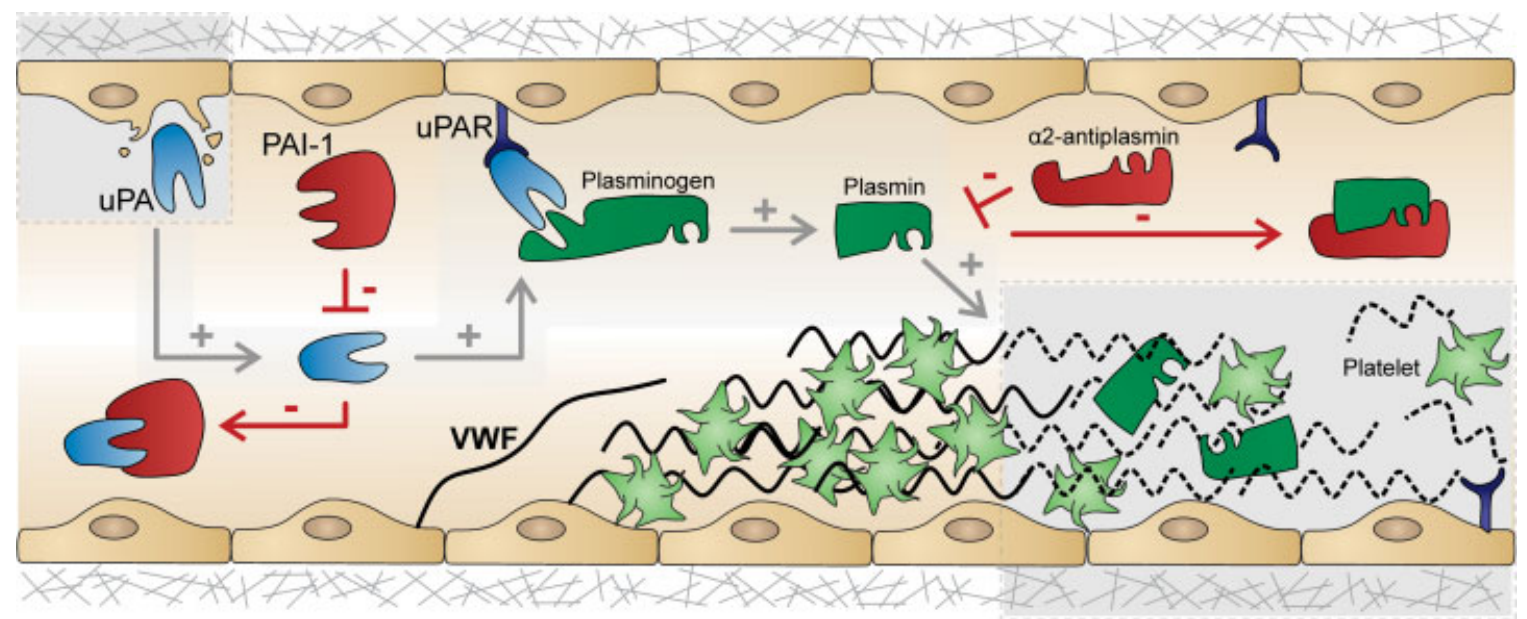

Fig. 1. Model: Plasminogen activation on endothelial cells mediates degradation of platelet-VWF complexes. PAI-1, plasminogen activator inhibitor-1; uPA, urokinase-type plasminogen activator; UPAR, UPA receptor; VWF, von Willebrand factor. 
treatment of TTP patients with thrombolytic agents, especially as these patients are thrombocytopenic. This safety aspect is of critical importance, and should be completely and carefully investigated in a preclinical setting.

However, it is also very important to note that the main body of clinical experience on the relationship between thrombolysis and bleeding is based on the treatment of types of thrombosis that do clinically not resemble TTP at all. Actually, there is little to no clinical experience with thrombolytic agents in TTP. We would like to challenge the dogma that thrombolytic treatment of TTP will carry a high risk of bleeding: we believe that it will be possible to safely use thrombolytic agents for treatment of TTP.

- Dosing: It is logical to assume that the treatment regimen (dosage, length, and intensity) of thrombolytic therapy that will be required for TTP is the same as that is needed for other types of thrombosis. However, this may not be the case: we found that a single dose of the direct-acting plasminogen activator streptokinase was sufficient to attenuate symptoms of TTP in an ADAMTS13-deficient mouse model (at $\sim 20 \%$ of the loading dose by plasma concentration that is given for pulmonary embolism $[\mathrm{PE}])^{64}$ Prolonged continuous infusion of thrombolysis was not required (this is indicated for treatment of PE or deep vein thrombosis [DVT]). We found no macroscopic evidence for bleeding or a perturbed secondary hemostasis after this treatment. Dosing studies in various preclinical models will be helpful to estimate how much plasminogen activation is actually needed for the management of TTP. This is important as the incidence of bleeding events is directly related to the intensity of thrombolytic therapy. ${ }^{72}$ Only after the required dosage is established can any potential off-target effects of thrombolysis on primary and secondary hemostasis really be evaluated.

- Molecular mechanisms: microthrombi in TTP are VWFrich but fibrin-poor. Thrombi in other forms of thrombosis generally contain fibrin, which is protected from degradation by plasmin through a variety of molecular mechanisms. As a result, thrombus breakdown by plasmin is relatively slow and their destruction requires a prolonged exposure to therapeutic plasminogen activators. This treatment can lead to a partial consumption of fibrinogen by plasmin. For example, treatment of ischemic stroke patients with tPA leads to a $26 \%$ decrease in fibrinogen levels. This decrease is associated with an increased risk on thrombolysis-related bleeding, especially in patients with low fibrinogen levels prior to treatment. ${ }^{73}$ In comparison to "classic" breakdown of fibrin, plasmin-mediated destruction of platelet-VWF complexes occurs relatively rapid. ${ }^{68}$ Furthermore, VWF is not protected from cleavage by dedicated molecular mechanisms (as far as we know). These differences may strongly influence the amount of plasmin that is required for TTP treatment, as well as the risk on off-target effects.

- Patient characteristics: Age is a strong risk factor for the development of cardiovascular events. Moreover, age is a strong risk factor for thrombolysis-related bleeding in these patients. In contrast, TTP patients may develop attacks at much earlier times in life (with potential implications for bleeding risk). Furthermore, it is expected that TTP patients will not receive anticoagulant therapy (a strong risk factor for thrombolysis-related bleeding). Finally, thrombolysis-related bleeding generally occurs in larger vessels that were previously occluded (e.g., intracerebral hemorrhage follows after stroke). In contrast, patients with TTP experience thrombosis of the microvasculature, where blood flow, blood pressure, and vessel wall composition are strikingly different. Also, these determinants may influence the risk on thrombolysis-related bleeding.

- Consumptive thrombocytopenia-prohemostatic effects of antihemostatic agents: During thrombocytopenia in general, any treatment that further compromises hemostasis (either primary or secondary) can logically be expected to elicit bleeding problems. However, thrombocytopenia in TTP is the result of an ongoing consumptive reaction, in which platelets accumulate in microvessels and become unavailable for hemostasis. Any treatment that destroys microthrombi would "break this circle," thereby attenuating thrombocytopenia and reducing bleeding risk. For example, it was recently reported that full in vivo neutralization of VWF with nanobody ALX0681 did not induce bleeding in a primate model for acquired TTP during thrombocytopenia. ${ }^{13}$ This can be attributed to restoration of platelet counts (prohemostatic), despite full blockade of VWF by ALX-0681 (a potent antihemostatic agent). A clinical study with ALX-0681 in patients with TTP has recently confirmed these findings. ${ }^{16}$ In a similar manner, platelet counts normalized when we treated ADAMTS13-deficient mice in a mouse model for TTP with thrombolysis. This suggests that plasmin may exert a prohemostatic role in the consumptive thrombocytopenia that hallmarks TTP, and that this would eventually reduce the bleeding risk.

\section{The Optimal Thrombolytic Agent}

We expect that not all types of thrombolytic agents will be equally effective for the treatment of TTP. Direct-acting plasminogen activators may be useful because plasmin has a direct binding affinity for unfolded VWF. ${ }^{64}$ However, the immunogenic potential of these agents limits their attractiveness for repeated use in TTP patients (as they are of bacterial origin). The major clot-busting drug tPA can directly bind to fibrin, but it is unable to target plateletVWF complexes for degradation in vitro. ${ }^{64}$ In contrast, uPAtriggered plasminogen activation leads to rapid cleavage of VWF, but only in the presence of cell-surface expressed uPAR. Because uPA levels are reportedly reduced during TTP attacks, ${ }^{65}$ we believe that reinforcement of the urokinase system provides a viable target for further investigation as a therapy. Clinical experience with uPA is very limited but appears positive: a case-report describes that uPA was repeatedly used successfully for the treatment of TTP. $^{74}$ Ultimately, it may be attractive to develop a new type 
of thrombolytic agent (e.g., by development of an antibody fusion protein ${ }^{75}$ ) that directly targets VWF-rich microthrombi.

\section{Conclusion}

ADAMTS13 is a key regulator of VWF and its deficiency is strongly linked to TTP. However, the absence or presence of ADAMTS13 alone does not fully explain the clinical picture of this condition. This literature overview shows that auxiliary mechanisms are in place to keep VWF under control. We hypothesize that plasmin is an important endogenous regulator of the thrombogenicity of VWF and plays a role in the etiology of TTP. Plasminogen activation takes place on the endothelium near sites of vascular obstruction. We propose that stimulation or reinforcement of plasminogen activation (in conjunction with other treatments) has therapeutic value for the treatment of TTP.

\section{Acknowledgments}

CT is a Postdoctoral Fellow supported by the Research Foundation-Flanders (FWO), Belgium. CM is supported by A Veni Fellowship (016-.-126-.-159), provided by the Netherlands Organization For Scientific Research (NWO). We thank Louis Deforche for creating the figure.

\section{References}

1 Springer TA. von Willebrand factor, Jedi knight of the bloodstream. Blood 2014;124(9):1412-1425

2 George JN, Nester CM. Syndromes of thrombotic microangiopathy. N Engl J Med 2014;371(19):1847-1848

3 Furlan M, Robles R, Lämmle B. Partial purification and characterization of a protease from human plasma cleaving von Willebrand factor to fragments produced by in vivo proteolysis. Blood 1996; 87(10):4223-4234

4 Tsai HM. Physiologic cleavage of von Willebrand factor by a plasma protease is dependent on its conformation and requires calcium ion. Blood 1996;87(10):4235-4244

5 Gerritsen HE, Robles R, Lämmle B, Furlan M. Partial amino acid sequence of purified von Willebrand factor-cleaving protease. Blood 2001;98(6):1654-1661

6 Fujikawa K, Suzuki H, McMullen B, Chung D. Purification of human von Willebrand factor-cleaving protease and its identification as a new member of the metalloproteinase family. Blood 2001;98(6): $1662-1666$

7 Crawley JTB, de Groot R, Xiang Y, Luken BM, Lane DA. Unraveling the scissile bond: how ADAMTS13 recognizes and cleaves von Willebrand factor. Blood 2011;118(12):3212-3221

8 Tersteeg C, Schiviz A, De Meyer SF, et al. Potential for recombinant ADAMTS13 as an effective therapy for acquired thrombotic thrombocytopenic purpura. Arterioscler Thromb Vasc Biol 2015; DOI: ATVBAHA.115.306014.

9 Schiviz A, Wuersch K, Piskernik C, et al. A new mouse model mimicking thrombotic thrombocytopenic purpura: correction of symptoms by recombinant human ADAMTS13. Blood 2012; 119(25):6128-6135

10 Jian C, Xiao J, Gong L, et al. Gain-of-function ADAMTS13 variants that are resistant to autoantibodies against ADAMTS13 in patients with acquired thrombotic thrombocytopenic purpura. Blood 2012;119(16):3836-3843
11 Li GW, Rambally S, Kamboj J, et al. Treatment of refractory thrombotic thrombocytopenic purpura with $\mathrm{N}$-acetylcysteine: a case report. Transfusion 2014;54(5):1221-1224

12 Shortt J, Opat SS, Wood EM. N-Acetylcysteine for thrombotic thrombocytopenic purpura: is a von Willebrand factor-inhibitory dose feasible in vivo? Transfusion 2014;54(9):2362-2363

13 Callewaert F, Roodt J, Ulrichts H, et al. Evaluation of efficacy and safety of the anti-VWF Nanobody ALX-0681 in a preclinical baboon model of acquired thrombotic thrombocytopenic purpura. Blood 2012;120(17):3603-3610

14 Feys HB, Roodt J, Vandeputte N, et al. Inhibition of von Willebrand factor-platelet glycoprotein Ib interaction prevents and reverses symptoms of acute acquired thrombotic thrombocytopenic purpura in baboons. Blood 2012;120(17):3611-3614

15 Holz JB. The TITAN trial-assessing the efficacy and safety of an anti-von Willebrand factor nanobody in patients with acquired thrombotic thrombocytopenic purpura. Transfus Apheresis Sci 2012;46(3):343-346

16 Peyvandi F, Scully M, Knobl P, et al. Additional data from the titan trial with the anti-VWF nanobody caplacizumab in the treatment of acquired TTP. J Thromb Haemost 2015;13(2):LB006

17 Tsai HM, Lian EC. Antibodies to von Willebrand factor-cleaving protease in acute thrombotic thrombocytopenic purpura. $\mathrm{N}$ Engl J Med 1998;339(22):1585-1594

18 Rieger M, Mannucci PM, Kremer Hovinga JA, et al. ADAMTS13 autoantibodies in patients with thrombotic microangiopathies and other immunomediated diseases. Blood 2005;106(4):1262-1267

19 Lotta LA, Wu HM, Mackie IJ, et al. Residual plasmatic activity of ADAMTS13 is correlated with phenotype severity in congenital thrombotic thrombocytopenic purpura. Blood 2012;120(2):440-448

20 Furlan M, Lämmle B. Aetiology and pathogenesis of thrombotic thrombocytopenic purpura and haemolytic uraemic syndrome: the role of von Willebrand factor-cleaving protease. Best Pract Res Clin Haematol 2001;14(2):437-454

21 Kremer Hovinga JA, Vesely SK, Terrell DR, Lämmle B, George JN. Survival and relapse in patients with thrombotic thrombocytopenic purpura. Blood 2010;115(8):1500-1511, quiz 1662

22 Peyvandi F, Lavoretano S, Palla R, et al. ADAMTS13 and antiADAMTS13 antibodies as markers for recurrence of acquired thrombotic thrombocytopenic purpura during remission. Haematologica 2008;93(2):232-239

23 Motto DG, Chauhan AK, Zhu G, et al. Shigatoxin triggers thrombotic thrombocytopenic purpura in genetically susceptible ADAMTS13-deficient mice. J Clin Invest 2005;115(10):2752-2761

24 Picard C, Burtey S, Bornet C, Curti C, Montana M, Vanelle P. Pathophysiology and treatment of typical and atypical hemolytic uremic syndrome. Pathol Biol (Paris) 2015;63(3):136-143

25 Tsai HM. Pathophysiology of thrombotic thrombocytopenic purpura. Int J Hematol 2010;91(1):1-19

26 Fuchs TA, Kremer Hovinga JA, Schatzberg D, Wagner DD, Lämmle B. Circulating DNA and myeloperoxidase indicate disease activity in patients with thrombotic microangiopathies. Blood 2012; 120(6):1157-1164

27 Brinkmann V, Reichard U, Goosmann C, et al. Neutrophil extracellular traps kill bacteria. Science 2004;303(5663):1532-1535

28 Clark SR, Ma AC, Tavener SA, et al. Platelet TLR4 activates neutrophil extracellular traps to ensnare bacteria in septic blood. Nat Med 2007;13(4):463-469

29 Fuchs TA, Brill A, Wagner DD. Neutrophil extracellular trap (NET) impact on deep vein thrombosis. Arterioscler Thromb Vasc Biol 2012;32(8):1777-1783

30 Demers M, Krause DS, Schatzberg D, et al. Cancers predispose neutrophils to release extracellular DNA traps that contribute to cancer-associated thrombosis. Proc Natl Acad Sci U S A 2012; 109(32):13076-13081

31 Thomas GM, Carbo C, Curtis BR, et al. Extracellular DNA traps are associated with the pathogenesis of TRALI in humans and mice. Blood 2012;119(26):6335-6343 
32 Chen G, Zhang D, Fuchs TA, Manwani D, Wagner DD, Frenette PS. Heme-induced neutrophil extracellular traps contribute to the pathogenesis of sickle cell disease. Blood 2014;123(24): 3818-3827

33 Borissoff JI, Joosen IA, Versteylen MO, et al. Elevated levels of circulating DNA and chromatin are independently associated with severe coronary atherosclerosis and a prothrombotic state. Arterioscler Thromb Vasc Biol 2013;33(8):2032-2040

34 Fuchs TA, Alvarez JJ, Martinod K, Bhandari AA, Kaufman RM, Wagner DD. Neutrophils release extracellular DNA traps during storage of red blood cell units. Transfusion 2013;53(12):3210-3216

35 von Brühl ML, Stark K, Steinhart A, et al. Monocytes, neutrophils, and platelets cooperate to initiate and propagate venous thrombosis in mice in vivo. J Exp Med 2012;209(4):819-835

36 Maga TK, Nishimura CJ, Weaver AE, Frees KL, Smith RJ. Mutations in alternative pathway complement proteins in American patients with atypical hemolytic uremic syndrome. Hum Mutat 2010; 31(6):E1445-E1460

37 Zipfel PF, Edey M, Heinen S, et al. Deletion of complement factor Hrelated genes CFHR1 and CFHR3 is associated with atypical hemolytic uremic syndrome. PLoS Genet 2007;3(3):e41

38 Turner N, Nolasco L, Nolasco J, Sartain S, Moake J. Thrombotic microangiopathies and the linkage between von Willebrand factor and the alternative complement pathway. Semin Thromb Hemost 2014;40(5):544-550

39 Feng S, Liang X, Cruz MA, et al. The interaction between factor $\mathrm{H}$ and von Willebrand factor. PLoS ONE 2013;8(8):e73715

40 Rayes J, Roumenina LT, Dimitrov JD, et al. The interaction between factor $\mathrm{H}$ and VWF increases factor $\mathrm{H}$ cofactor activity and regulates VWF prothrombotic status. Blood 2014;123(1): 121-125

41 Nolasco L, Nolasco J, Feng S, Afshar-Kharghan V, Moake J. Human complement factor $\mathrm{H}$ is a reductase for large soluble von Willebrand factor multimers-brief report. Arterioscler Thromb Vasc Biol 2013;33(11):2524-2528

42 Wohner N, Kovács A, Machovich R, Kolev K. Modulation of the von Willebrand factor-dependent platelet adhesion through alternative proteolytic pathways. Thromb Res 2012;129(4):e41-e46

43 Raife TJ, Cao W, Atkinson BS, et al. Leukocyte proteases cleave von Willebrand factor at or near the ADAMTS13 cleavage site. Blood 2009;114(8):1666-1674

44 Hollestelle MJ, Sprong T, Bovenschen N, et al. von Willebrand factor activation, granzyme-B and thrombocytopenia in meningococcal disease. J Thromb Haemost 2010;8(5):1098-1106

45 Kokame K, Nobe Y, Kokubo Y, Okayama A, Miyata T. FRETS-VWF73, a first fluorogenic substrate for ADAMTS13 assay. $\mathrm{Br} \mathrm{J}$ Haematol 2005;129(1):93-100

46 Mikes B, Sinkovits G, Farkas P, et al. Elevated plasma neutrophil elastase concentration is associated with disease activity in patients with thrombotic thrombocytopenic purpura. Thromb Res 2014;133(4):616-621

47 Budde U, Schneppenheim R. Interactions of von Willebrand factor and ADAMTS13 in von Willebrand disease and thrombotic thrombocytopenic purpura. Hamostaseologie 2014;34(3): 215-225

48 Buzza MS, Dyson JM, Choi H, et al. Antihemostatic activity of human granzyme B mediated by cleavage of von Willebrand factor. J Biol Chem 2008;283(33):22498-22504

49 Hollestelle MJ, Lai KW, van Deuren M, et al. Cleavage of von Willebrand factor by granzyme M destroys its factor VIII binding capacity. PLoS ONE 2011;6(9):e24216

50 Wensink AC, Hack CE, Bovenschen N. Granzymes regulate proinflammatory cytokine responses. J Immunol 2015;194(2):491-497

51 Chandler WL, Jelacic S, Boster DR, et al. Prothrombotic coagulation abnormalities preceding the hemolytic-uremic syndrome. N Engl J Med 2002;346(1):23-32

52 Monteagudo J, Pereira A, Reverter JC, et al. Thrombin generation and fibrinolysis in the thrombotic thrombocytopenic purpura and the hemolytic-uremic syndrome. Thromb Haemost 1991;66(5): 515-519

53 Raife T, Friedman KD, Fenwick B. Lepirudin prevents lethal effects of Shiga toxin in a canine model. Thromb Haemost 2004;92(2): 387-393

54 Wada H, Mori Y, Shimura M, et al. Poor outcome in disseminated intravascular coagulation or thrombotic thrombocytopenic purpura patients with severe vascular endothelial cell injuries. Am J Hematol 1998;58(3):189-194

55 Sagripanti A, Carpi A, Baicchi U, et al. Plasmatic parameters of coagulation activation in thrombotic microangiopathy. Biomed Pharmacother 1996;50(8):357-362

56 Takahashi H, Tatewaki W, Wada K, Shibata A. Thrombin generation in patients with thrombotic thrombocytopenic purpura. Am J Hematol 1989;32(4):255-257

57 Hoylaerts M, Rijken DC, Lijnen HR, Collen D. Kinetics of the activation of plasminogen by human tissue plasminogen activator. Role of fibrin. J Biol Chem 1982;257(6):2912-2919

58 Ellis V, Behrendt N, Danø K. Plasminogen activation by receptorbound urokinase. A kinetic study with both cell-associated and isolated receptor. J Biol Chem 1991;266(19):12752-12758

59 Miles LA, Parmer RJ. Plasminogen receptors: the first quarter century. Semin Thromb Hemost 2013;39(4):329-337

60 Hamilton KK, Fretto LJ, Grierson DS, McKee PA. Effects of plasmin on von Willebrand factor multimers. Degradation in vitro and stimulation of release in vivo. J Clin Invest 1985;76(1): 261-270

61 Federici AB, Berkowitz SD, Zimmerman TS, Mannucci PM. Proteolysis of von Willebrand factor after thrombolytic therapy in patients with acute myocardial infarction. Blood 1992;79(1): 38-44

62 Feys HB, Vandeputte N, Palla R, et al. Inactivation of ADAMTS13 by plasmin as a potential cause of thrombotic thrombocytopenic purpura. J Thromb Haemost 2010;8(9):2053-2062

63 Crawley JTB, Lam JK, Rance JB, Mollica LR, O'Donnell JS, Lane DA. Proteolytic inactivation of ADAMTS13 by thrombin and plasmin. Blood 2005;105(3):1085-1093

64 Tersteeg C, de Maat S, De Meyer SF, et al. Plasmin cleavage of von Willebrand factor as an emergency bypass for ADAMTS13 deficiency in thrombotic microangiopathy. Circulation 2014;129(12): 1320-1331

65 Kakishita E, Koyama T, Higuchi M, Kunitomi O, Oura Y, Nagai K. Fibrinogenolysis in thrombotic thrombocytopenic purpura. Am J Hematol 1989;32(1):14-19

66 Hoirisch-Clapauch S, Nardi AE. A role for tissue plasminogen activator in thrombotic thrombocytopenic purpura. Med Hypotheses 2014;83(6):747-750

67 Kroon ME, Koolwijk P, van der Vecht B, van Hinsbergh VW. Urokinase receptor expression on human microvascular endothelial cells is increased by hypoxia: implications for capillarylike tube formation in a fibrin matrix. Blood 2000;96(8): 2775-2783

68 Graham CH, Fitzpatrick TE, McCrae KR. Hypoxia stimulates urokinase receptor expression through a heme protein-dependent pathway. Blood 1998;91(9):3300-3307

69 Xu Y, Hagege J, Mougenot B, Sraer JD, Rønne E, Rondeau E. Different expression of the plasminogen activation system in renal thrombotic microangiopathy and the normal human kidney. Kidney Int 1996;50(6):2011-2019

70 Bu F, Maga T, Meyer NC, et al. Comprehensive genetic analysis of complement and coagulation genes in atypical hemolytic uremic syndrome. J Am Soc Nephrol 2014;25(1):55-64

71 Hugenholtz GCG, Lisman T. Letter by Hugenholtz and Lisman regarding article, "plasmin cleavage of von Willebrand factor as an emergency bypass for ADAMTS13 deficiency in thrombotic microangiopathy.". Circulation 2015;131(2):e18

72 Sinnaeve PR, Armstrong PW, Gershlick AH, et al; STREAM investigators. ST-segment-elevation myocardial infarction 
patients randomized to a pharmaco-invasive strategy or primary percutaneous coronary intervention: Strategic Reperfusion Early After Myocardial Infarction (STREAM) 1-year mortality follow-up. Circulation 2014;130(14):1139-1145

73 Vandelli L, Marietta M, Gambini M, et al. Fibrinogen decrease after intravenous thrombolysis in ischemic stroke patients is a risk factor for intracerebral hemorrhage. J Stroke Cerebrovasc Dis 2015;24(2):394-400
74 Vesconi S, Langer M, Rossi E, Donati MB. Urokinase treatment for severe neurological complications in a patient with thrombotic thrombocytopenic purpura. Haemostasis 1981;10(5):289-295

75 Wang X, Palasubramaniam J, Gkanatsas Y, et al. Towards effective and safe thrombolysis and thromboprophylaxis: preclinical testing of a novel antibody-targeted recombinant plasminogen activator directed against activated platelets. Circ Res 2014; 114(7):1083-1093 